\title{
Talavera-Ibarra, A. y Vega-Ramírez, A. de la (2016). Library Education in Peru: Historical and Future Perspectives. En M. Seadle, C. M. Chu, U. Stöckel y B. Crumpton (Eds.), Educating the Profession: 40 years of the IFLA Section on Education and Training (pp. 191-215). Berlín, Alemania: Walter de Gruyter GmbH \& Co KG.
}

La IFLA (International Federation Library Association) es un organismo que agrupa a las asociaciones de bibliotecarios de todo el mundo. Recientemente ha publicado un libro por los 40 años de la Sección de Educación y Formación de ese organismo donde se incluyen colaboraciones relacionadas con el cambio y desarrollo producidos en la profesión de bibliotecario en las últimas décadas. El texto examina tanto el pasado así como las tendencias futuras de los estudios en las escuelas de Bibliotecología y Ciencias de la Información en Europa, Asia, África, Australia y las Américas.

Es satisfactorio reconocer que la investigación histórica y la relativa al estado de la cuestión en la Bibliotecología se fortalecen actualmente en el Perú. Hay trabajos pioneros sobre la Escuela Nacional de Bibliotecarios que su propio fundador incluye en sus memorias (Basadre, 1975); posteriormente se publican dos textos que plantean un análisis histórico de la trascendencia de esta escuela (Mac Kee de Maurial, 1963; Pardo Sandoval, 1990). Otros autores investigaron a Jorge Basadre y su labor como bibliotecario (Castro Aliaga, 2012) o la importancia de algunas de las bibliotecas universitarias más importantes del país, como la de la Universidad Nacional Mayor de San Marcos-UNMSM (Cajas Rojas, 2008); mientras que la publicación de Corzo y Lumbreras (2003) hace un balance de los primeros 50 años de existencia de la Bibliotecología como profesión, así como Estrada-Cuzcano y Alfaro-Mendives (2016) centran su investigación en el rol de la UNMSM en la consolidación de la Bibliotecología peruana. Siguiendo esa línea aparece el trabajo de Talavera-Ibarra y Vega-Ramírez, ambas docentes de la especialidad en la Pontificia Universidad Católica del Perú (PUCP) y muestran un 
amplio panorama de la realidad actual de las dos escuelas de Bibliotecología existentes en el Perú: la de las universidades San Marcos y la de la Católica que reseñamos a continuación.

Se hace un análisis retrospectivo sobre el origen de la formación bibliotecaria en América Latina para luego hacer énfasis en nuestro país; parte de la histórica Escuela Nacional de Bibliotecarios, que surge a partir del incendio de la Biblioteca Nacional del Perú en 1943, para luego de casi cuatro décadas trasladarse a la UNMSM en 1980 y adquirir rango universitario como Programa de Bibliotecología y Ciencias de la Información. Se reseña también el surgimiento de la especialidad de Bibliotecología y Ciencias de la Información en la PUCP, a partir del año 1985; desde esa fecha no se han creado otras escuelas de la especialidad.

Se describe al detalle la formación profesional, los grados y títulos, la educación continua, la investigación y las publicaciones existentes en la disciplina. La formación profesional de ambas escuelas es expuesta detalladamente y determina los créditos académicos que se asignan a cada área en los planes de estudio; se demuestra que los porcentajes asignados a las áreas en ambas escuelas son parecidos; además, se agregan datos sobre el número de egresados, bachilleres y licenciados que hay por cada escuela, junto al número de docentes nombrados y contratados con sus respectivas categorías (auxiliar, asociado y principal). La comparación de las áreas de ambas escuelas se realiza a partir de la estructura establecida por el EDIBCIC (Asociación de Educación e Investigación en Ciencia de la Información de Iberoamérica y el Caribe) y se complementa con los criterios determinados por las escuelas de Bibliotecología del MERCOSUR; ello demuestra que ambas escuelas no distan mucho de las recomendaciones sugeridas en la propuesta de las autoras.

Con relación a la educación continua se incluyen las múltiples actividades que se realizan en el país, además de las universitarias; entre otros, resalta el Seminario de la Realidad Bibliotecológica llevado en cada año en la UNMSM. Se detallan las particularidades de la investigación especificando las características de la subvención que brindan las universidades a la investigación 
académica; asimismo, se toma como referencia algunos estudios sobre las tendencias existentes en la investigación universitaria, especialmente en las tesis de pregrado (licenciatura). Complementan el análisis con las publicaciones hechas por la comunidad investigadora de la UNMSM y de la PUCP en revistas de diversos países, pero reseñan también un número de publicaciones periódicas que no tuvieron impacto ni sostenibilidad en el país, haciendo evidente que no hay una revista en Bibliotecología posicionada en el mundo académico.

La parte final se refiere al mercado laboral y también se comentan trabajos realizados sobre la movilidad del empleo de los egresados en Bibliotecología y Ciencias de la Información.

En líneas generales, se muestra una visión global de lo acontecido en el Perú en la especialidad, por lo que esta investigación contribuye a establecer un registro de la memoria. La publicación en inglés de este libro puede ser una fortaleza o una debilidad porque está dirigido una comunidad investigadora diferente a la de habla española, aunque se supone que muchos investigadores manejan el inglés; otra dificultad es que el texto no es de acceso abierto, lo que podría ser una restricción para el acceso de los investigadores de nuestro medio. A modo de conclusión, las autoras invocan a formar profesionales que respondan a nuevos paradigmas surgidos de una sociedad de la información, especialmente en aspectos relacionados con el acceso a la información y al conocimiento con alto componente en el manejo de tecnologías de la información y comunicación; además, se propone la descentralización de los estudios de la especialidad fuera de la capital del país (Lima).

Alonso Estrada-Cuzcano, Universidad Nacional Mayor de San Marcos mestradac@unmsm.edu.pe

\section{Referencias bibliográficas}

Basadre, J. (1975). Recuerdos de un bibliotecario peruano: 1919-1930, 1930-1932, 1935-1942, 1943-1948, 1956-1958. Lima, Perú: Editorial Historia. 
Cajas Rojas, A. I. (2008). Historia de la Biblioteca Central de la Universidad de San Marcos: 1923 a 1966. (Tesis para optar por el grado de Magíster en Historia), Universidad Nacional Mayor de San Marcos. Facultad de C i e n cias Sociales, Lima. Re cuperado d e http://cybertesis.unmsm.edu.pe/xmlui/bitstr eam/handle/cybertesis/234 4/cajas_ra.pdf

Castro Aliaga, C. A. (2012). Aportes al estudio de la bibliotecología peruana: vida y obra de Jorge Basadre Grohmann (1903-1980). (Tesis para optar por el grado de Doctor en Ciencias de la Información), Universidad Complutense de M a d rid, $\quad$ M a d r i d. $\quad$ R e c u p e r a d o d e http://eprints.ucm.es/16180/1/T33827.pdf

Corzo, O. y Lumbreras, S. (2003). 50 años de enseñanza bibliotecológica 1943-1993. Lima, Perú: Universidad Nacional Mayor de San Marcos.

Estrada-Cuzcano, A. y Alfaro-Mendives, K. l. (2016). Presencia de la Universidad Nacional Mayor de San Marcos en la Bibliotecología Peruana: Estudio historiográfico. Letras, 87(125), 105-122.

Mac Kee de Maurial, N. (1963). Veinte años de existencia de la Escuela Nacional de Bibliotecarios. Boletín de la Biblioteca Nacional, 17(28), 8-11.

Pardo Sandoval, T. (1990). El primer cuarto de siglo de la Escuela Nacional de Bibliotecarios. Fénix: Revista de la Biblioteca Nacional (36-37), 60-124. 